\title{
An Overview of Various Importance Measures of Reliability System
}

\author{
Kalpesh P. Amrutkar, Kirtee K. Kamalja \\ Department of Statistics \\ School of Mathematical Sciences \\ North Maharashtra University, Jalgaon, India \\ *Corresponding author: amrutkarkp@gmail.com
}

(Received November 15, 2016; Accepted January 3, 2017)

\begin{abstract}
One of the purposes of system reliability analysis is to identify the weaknesses or the critical components in a system and to quantify the impact of component's failures. Various importance measures are being introduced by many researchers since 1969. These component importance measures provide a numerical rank to determine which components are more important to system reliability improvement or more critical to system failure. In this paper, we overview various components importance measures and briefly discuss them with examples. We also discuss some other extended importance measures and review the developments in study of various importance measures with respect to some of the popular reliability systems.
\end{abstract}

Keywords: Reliability systems. Importance measures

\section{Introduction}

Reliability evaluation has a vital importance at all stages of processing and controlling in engineering systems. Apart from reliability evaluation of the systems, the study of component importance is also an important part. The component importance (reliability importance index) is valuable in establishing the direction and prioritization of actions related to an upgrading effort (reliability improvement) in system design or suggesting the most effective way to operate and maintain system status. The main purpose of system reliability analysis is to identify the weaknesses/critical components in a system and to quantify the impact of component's failures. Since 1969, various component importance measures are being introduced by the researchers. These measures provide a numerical rank to determine which components are more important (more critical) to system reliability improvement (system failure).

In this paper, we overview various component importance measures for coherent systems. Bergman et al. (1985) provides an interesting survey on reliability theory. Boland and El-Neweihi (1995) overviewed the measures of component importance in binary coherent systems and suggested some new component importance measures which might be useful to analyze when the system is about to undergo a reliability improvement. Kuo and Zhu (2012a, 2012b, 2012c) surveyed the recent advances of importance measures in reliability and Zhu and Kuo (2014) gave the implications to mathematical programming, sensitivity and uncertainty analysis, probabilistic risk analysis and probabilistic safety assessment. The review of particular research topic usually helps the researchers in the sense of availability of all related literature in collective form. With this realization, we motivated to present the variety of importance measures altogether. The idea is to bring the importance measures with different bases/ backgrounds in collective form.

Birnbaum (1969) was first to introduce the concept of importance measures. He classified importance measures into three classes based on the knowledge needed for determining them. 
International Journal of Mathematical, Engineering and Management Sciences

Vol. 2, No. 3, 150-171, 2017

https://dx.doi.org/10.33889/IJMEMS.2017.2.3-014

These three classes are as; structure importance measures, reliability importance measures and lifetime importance measures. We briefly discuss these three classes of importance measures.

\section{(a) Structure Importance Measures}

Structure importance measures are defined under the assumption that the system structure is known. It measures the relative importance of various components with respect to their positions in a system. It is relevant to building a system when several components with distinct reliabilities can be arbitrarily assigned to several locations in the system. Presumably, one would like to assign the most reliable component to the more important location.

\section{(b) Reliability Importance Measures}

Reliability importance measures depend on both the system structure and reliability of components over an explicit and fixed mission time. It measures the change in the system reliability with respect to the change in reliability of a specific component.

\section{(c) Lifetime Importance Measures}

The lifetime importance measures, depends on both, the position of the component in the system and component lifetime distribution. Kuo and Zhu (2012c) classified the lifetime importance measures into two classes as Time-Dependent Lifetime (TDL) importance and Time Independent Lifetime (TIL) importance, depending on whether they are a function of time.

In this paper, we overview the various importance measures based on the above three classes and also discuss some other extended importance measures. The paper is organized as follows.

In Section 2, we discuss various structure importance measures. Section 3 covers the probability based importance measures and presents examples for some of the popular systems. Section 4 includes a review of lifetime importance measures. In section 5, we overview some other importance measures studied in the literature. Section 6, includes introduction to some extended importance measures. Section 7, reviews the study of importance measures of some weighted systems. Finally, Section 8 concludes the overview of importance measures.

\section{Structure Importance Measures}

In this section, we discuss some structure importance measures, which actually evaluate the relative importance of various components with respect to their positions in a system. The component structure importance generally represents the importance of the positions in the system that the components occupy. The structure importance measure is used to assign a rank to the components when the component reliabilities are not available. We discuss some of the structure importance measures in the following.

Consider a $n$-component binary coherent system with structure function $\phi($.$) . Let x_{i}$ indicates a state (failed/working) of the $i^{\text {th }}$ component of the system. That is,

$$
\begin{aligned}
& x_{i}= \begin{cases}1 & \text { if } i^{t h} \text { component is working } \\
0 \quad & \text { if } i^{t h} \text { component is failed. }\end{cases} \\
& \bar{p}_{i} \quad=\left(x_{1}, x_{2}, \ldots, x_{n}\right): \text { the vector of states of } n \text { components. }
\end{aligned}
$$


International Journal of Mathematical, Engineering and Management Sciences

Vol. 2, No. 3, 150-171, 2017

https://dx.doi.org/10.33889/IJMEMS.2017.2.3-014

$\underline{p} \quad=\left(p_{1}, p_{2}, \ldots, p_{n}\right):$ the vector of reliabilities of $n$-components.

$\bar{\phi}(\underline{x})$ the system state which is defined as, $\phi(\underline{x})=1(0)$ if the system is working (failed).

$R(\underline{p}) \quad$ system reliability with component reliability vector $\underline{p}$.

\section{(a) Birnbaum Structure (B-Structure) Importance}

A structure importance measure is introduced by Birnbaum (1969). The component structure importance generally represents the importance of the positions in the system that the components occupy.

Birnbaum (1969) defines the B-structure importance $I_{B}^{i}(\phi)$ of component $i$ as,

$I_{B}^{i}(\phi)=\sum_{\underline{x}}\left[\phi\left(1_{i}, \underline{x}\right)-\phi\left(0_{i}, \underline{x}\right)\right]$

where $\left(1_{i}, \underline{x}\right) \quad: \underline{x}$ with $x_{i}=1$

$\left(0_{i}, \underline{x}\right) \quad: \underline{x}$ with $x_{i}=0$

$\phi\left(1_{i}, \underline{x}\right) \quad:$ System state when $i^{\text {th }}$ component is working

$\phi\left(0_{i}, \underline{x}\right) \quad$ : System state when $i^{\text {th }}$ component is fail.

This formula further simplifies as follows.

$$
\begin{aligned}
& I_{B}^{i}(\phi)=R\left(\frac{1}{2}, \frac{1}{2}, \ldots \frac{1}{2}, 1_{i}, \frac{1}{2}, \ldots, \frac{1}{2}\right)-R\left(\frac{1}{2}, \frac{1}{2}, \ldots \frac{1}{2}, 0_{i}, \frac{1}{2}, \ldots, \frac{1}{2}\right) \\
& =R\left(1_{i}, \underline{p}\right)-R\left(0_{i}, \underline{p}\right) \\
& \text { where }\left(1_{i}, \underline{p}\right) \quad: \underline{p} \text { with } p_{i}=1 \text { and } p_{j}=\frac{1}{2}, j=1,2, \ldots, n ; j \neq i \\
& \left(0_{i}, \underline{p}\right) \quad: \underline{p} \text { with } p_{i}=0 \text { and } p_{j}=\frac{1}{2}, j=1,2, \ldots, n ; j \neq i \\
& R\left(1_{i}, \underline{p}\right) \quad: \text { System reliability when } i^{\text {th }} \text { component is working } \\
& R\left(0_{i}, \underline{p}\right) \quad: \text { System reliability when } i^{\text {th }} \text { component failed. }
\end{aligned}
$$

The B-structural importance establishes the probability of the system failure due to a given component when all components are assumed to be equally reliable i.e. each component has reliability $\frac{1}{2}$.

\section{(b) Fassell-Vesely (FV) Structure Importance}

The FV-importance is introduced by Fussell and Vesely (1972) which is based on the cut set and path set. The FV-importance is classified into $c$-type and $p$-type FV-importance. The $c$-type FVimportance, referred to as $c$-FV importance, is based on cut set while the $p$-type FV-importance, referred to as $p$-FV importance, is based on the path set of the system. Let $N=\{1,2, \ldots, n\}$ be a set of components of system.

The $c$-FV importance of component $i$ is denoted by $I_{F V^{c}}^{i}(\phi)$ and defined as,

$I_{F V^{c}}^{i}(\phi)=\frac{\mid\left\{\underline{x}: \exists C \in \overline{\mathcal{C}}_{i} \text { such that } C \subseteq N_{0}(\underline{x}) ; \phi(\underline{x})=0\right\} \mid}{|\{\underline{x}: \phi(\underline{x})=0\}|}$ 
International Journal of Mathematical, Engineering and Management Sciences

Vol. 2, No. 3, 150-171, 2017

https://dx.doi.org/10.33889/IJMEMS.2017.2.3-014

$$
=\frac{\mid\left\{\left(0_{i}, \underline{x}\right): \exists C \in \overline{\mathcal{C}}_{i} \text { such that } C \subseteq N_{0}\left(\left(0_{i}, \underline{x}\right)\right)\right\} \mid}{|\mathcal{C}|} .
$$

where $\quad N_{0}(\underline{x})=\left\{i \in N \mid x_{i}=0\right\} \quad$ : a cut set associated with a cut vector $\underline{x}$

$$
\begin{array}{ll}
\overline{\mathcal{P}} & : \text { set of minimal path vectors } \\
\overline{\mathcal{P}}_{i} & : \text { the minimal paths vectors containing component } i \\
P & : \text { minimal path from minimal path set } \overline{\mathcal{P}}
\end{array}
$$

Similarly, the $p$-FV structure importance of component $i$ is denoted by $I_{F V}^{i}(\phi)$ and is defined as,

$$
\begin{aligned}
I_{F V}^{i} p(\phi)= & \frac{\mid\left\{\underline{x}: \exists P \in \overline{\mathcal{P}}_{i} \text { such that } P \subseteq N_{1}(\underline{x}) ; \phi(\underline{x})=1\right\} \mid}{|\{\underline{x}: \phi(\underline{x})=1\}|} \\
= & \frac{\mid\left\{\left(1_{i}, \underline{x}\right): \exists P \in \overline{\mathcal{P}}_{i} \text { such that } P \subseteq N_{1}\left(\left(1_{i}, \underline{x}\right)\right)\right\} \mid}{|\mathcal{P}|} . \\
\text { where } & \begin{array}{ll}
N_{1}(\underline{x})=\left\{i \in N \mid x_{i}=1\right\} \quad: N_{1}(\underline{x}) \text { is a path set associated with path vector } \underline{x} \\
\overline{\mathcal{C}} & : \text { set of minimal cut vector } \\
& \overline{\mathcal{C}}_{i} \\
C & : \text { the minimal cut vector containing component } i
\end{array}
\end{aligned}
$$

\section{(c) Barlow-Proschan (BP) Structure Importance}

The BP-structure importance is introduced by Barlow and Proschan (1975). The BP-structure importance of component $i$ is denoted by $I_{B P}^{i}(\phi)$ and defined as,

$I_{B P}^{i}(\phi)=\int_{0}^{1}\left[R\left(1_{i}, \underline{p}\right)-R\left(0_{i}, \underline{p}\right)\right] d p$.

The BP-structure importance can be expressed in terms of B-structural importance as follows.

$$
I_{B P}^{i}(\phi)=\int_{0}^{1} I_{B}^{i}(\phi) d p
$$

\section{- Comparison between $I_{B}^{i}(\phi)$ and $I_{B P}^{i}(\phi)$}

The Birnbaum structure importance measure assumes the reliability of each component to be $\frac{1}{2}$ i.e. $p_{i}=\frac{1}{2}, i=1,2, \ldots, n$ while the BP-structure importance is average of $I_{B}^{i}(\phi)$ over all $p \in[0,1]$. Thus, both measures favor neither high nor low component reliabilities.

\section{(d) Cut Importance and Path Importance}

Butler (1979) proposes the two structure importances, namely, cut importance and path importance. The cut importance is a complete ranking of all components relative to their importance to the system and is defined on the basis of minimal cut sets while the path importance is defined on the basis of the minimal path set. The additional coverage of cut and path importances is discussed by Kuo and Zhu (2012c). 
International Journal of Mathematical, Engineering and Management Sciences

Vol. 2, No. 3, 150-171, 2017

https://dx.doi.org/10.33889/IJMEMS.2017.2.3-014

\section{(e) Permutation Importance and Permutation Equivalence}

The permutation importance and permutation equivalence measures are introduced by Boland et al. (1989). The definition of the permutation importance is based on the minimal cut set and minimal path set.

Component $i$ is more permutation important than component $j$ (denoted by $i>_{p e} j$ ) for structure function $\phi$ if

$\phi\left(1_{i}, 0_{j}, \underline{x}^{(i, j)}\right) \geq \phi\left(0_{i}, 1_{j}, \underline{x}^{(i, j)}\right) \quad$ holds for all $\underline{x}^{(i, j)}$

where, $\underline{x}^{(i, j)}=\left(x_{1}, x_{2}, \ldots, x_{i-1}, x_{i+1}, \ldots, x_{j-1}, x_{j+1}, \ldots, x_{n}\right)$.

That is, $\underline{x}^{(i, j)}$ is a vector of $n-2$ component states excluding $i^{\text {th }}$ and $j^{\text {th }}$ component.

If equality holds for some $\underline{x}^{(i, j)}$, then component $i$ and component $j$ are said to be permutation equivalent and is denoted by, $i={ }_{p e} j$.

Component $i$ and component $j$ are said to be permutation equivalent if and only if they are structurally symmetric and $\phi(\underline{x})$ is said to be a permutation symmetric in $x_{i}$ and $x_{j}$. Koutras et al. (1994) proved the following properties for permutation importance and permutation equivalence.

(i) If $i>_{p e} j$ and $j>_{p e} k$ then $i>_{p e} k$ (transitivity property).

(ii) If $i>_{p e} j$ for system structure $\phi$, then $i>_{p e} j$ for its dual system structure $\phi^{D}$ (dual of $\phi$ ) and vice versa (dual relation).

(iii) If component $i$ is in series (parallel) with the rest of the system, then

$$
\phi\left(0_{i}, 1_{j}, \underline{x}^{(i, j)}\right)=0 ; \phi\left(1_{i}, 0_{j}, \underline{x}^{(i, j)}\right)=1 \text {, for all } \underline{x}^{(i, j)} .
$$

This implies that component $i$ is more permutation important than component $j$ i.e. $i \geq_{p e} j$, for all $i \neq j$ (special case property).

In next section, we discuss the various reliability based importance measures.

\section{Reliability Importance Measures}

Reliability importance measures depend on both the system structure and reliability of components over an explicit and fixed mission time. It measures the change in the system reliability with respect to the change in reliability of a specific component.

In this section, we discuss the reliability importance measures which are considered when the mission time of a system is implicit and fixed, and consequently, the components are evaluated by their reliability at a fixed time point, i.e. the probability that component functions properly during the mission time (Kuo and Zhu, 2012c).

\section{(a) Birnbaum Reliability Importance}

Birnbaum reliability importance (B-importance) measure is introduced by Birnbaum (1969). It is used when we are provided the survival probabilities of all the $n$ components in a coherent system. It is the rate of increase in the system reliability with respect to the increase in component reliability. 
International Journal of Mathematical, Engineering and Management Sciences

Vol. 2, No. 3, 150-171, 2017

https://dx.doi.org/10.33889/IJMEMS.2017.2.3-014

Birnbaum (1969) defines the B-importance of system with component reliabilities $\underline{p}=$ $\left(p_{1}, p_{2}, \ldots, p_{n}\right)$ through the following.

The B-importance of $i^{\text {th }}$ component for the functioning of system is denoted by $I_{B S}^{i}\left(1_{i}, \underline{p}\right)$ and defined as,

$I_{B S}^{i}\left(1_{i}, \underline{p}\right)=P\left\{\phi(\underline{x})=1 \mid X_{i}=1\right\}-P\{\phi(\underline{x})=1\}$.

The B-importance of $i^{t h}$ component for the failure of system is denoted by $I_{B f}^{i}\left(0_{i}, \underline{p}\right)$ and defined as,

$I_{B f}^{i}\left(0_{i}, \underline{p}\right)=P\left\{\phi(\underline{x})=0 \mid X_{i}=0\right\}-P\{\phi(\underline{x})=0\}$.

Further Birnbaum (1969) defines the B-importance $I_{B}^{i}(p)$ of $i^{\text {th }}$ component in $n$ component system as addition of B-importance for system functioning and system failure. Thus $I_{B}^{i}(\underline{p})$ can be given as,

$$
\begin{aligned}
I_{B}^{i}(\underline{p}) & =I_{B S}^{i}\left(1_{i}, \underline{p}\right)+I_{B f}^{i}\left(0_{i}, \underline{p}\right) \\
& =P\left\{\phi(\underline{x})=1 \mid X_{i}=1\right\}-P\{\phi(\underline{x})=1\}+P\left\{\phi(\underline{x})=0 \mid X_{i}=0\right\}-P\{\phi(\underline{x})=0\} \\
& =P\left\{\phi(\underline{x})=1 \mid X_{i}=1\right\}+P\left\{\phi(\underline{x})=0 \mid X_{i}=0\right\}-1 \\
& =P\left\{\phi(\underline{x})=1 \mid X_{i}=1\right\}-\left[1-P\left\{\phi(\underline{x})=0 \mid X_{i}=0\right\}\right] \\
& =P\left\{\phi(\underline{x})=1 \mid X_{i}=1\right\}-P\left\{\phi(\underline{x})=1 \mid X_{i}=0\right\} .
\end{aligned}
$$

An equivalent definition of B-importance is given by Barlow and Proschan (1975).

$$
\begin{aligned}
I_{B}^{i}(\underline{p}) & =E\left(\phi\left(1_{i}, \underline{x}\right)-\phi\left(0_{i}, \underline{x}\right)\right) \\
& =R\left(1_{i}, \underline{p}\right)-R\left(0_{i}, \underline{p}\right)=\frac{\partial R(\underline{p})}{\partial p_{i}}
\end{aligned}
$$

$$
\begin{array}{cl}
\text { where }\left(1_{i}, \underline{p}\right)\left(\left(0_{i}, \underline{p}\right)\right) & \text { is } \underline{p} \text { with } p_{i}=1(0), \\
\phi\left(1_{i}, \underline{x}\right)\left(\phi\left(0_{i}, \underline{x}\right)\right) & \text { is a structure function with } i^{\text {th }} \text { component working (failed), } \\
R\left(1_{i}, \underline{p}\right)\left(R\left(0_{i}, \underline{p}\right)\right) & \text { is a reliability when } i^{\text {th }} \text { component is working (failed). }
\end{array}
$$

Specifically, (3.1) is given under the assumption that component reliabilities are independent.

Birnbaum measure is thus obtained by partial differentiation of the system reliability with respect to $p_{i}$ when the component reliabilities are independent. Chang et al. (2002) refers this measure as a Combinatorial Birnbaum Importance. According to the equivalent definition $(3.1), I_{B}^{i}(\underline{p})$ is a rate at which system reliability improves, when, the reliability of component $i$ improves. 
International Journal of Mathematical, Engineering and Management Sciences

Vol. 2, No. 3, 150-171, 2017

https://dx.doi.org/10.33889/IJMEMS.2017.2.3-014

- Observe that, the B-structure importance is a special case of B-importance with $p_{i}=\frac{1}{2}$, $i=1,2, \ldots, n$.

i.e. $\quad I_{B}^{i}(\phi)=I_{B}^{i}\left(\frac{1}{2}, \frac{1}{2}, \ldots, \frac{1}{2}\right)$.

- The B-importance is referred as marginal reliability importance by Hong and Lie (1993), Armstrong (1995), Hsu and Yuang (1999), Lu and Jiang (2007), Gao et al. (2007).

B-importance is the most popular importance measure. We demonstrate the evaluation of Bimportance for series, parallel and $k$-out-of- $n$ systems through following examples.

Example 1: A series system works (fails) if and only if all (at least one) components works (fail). The structure function $\phi(\underline{x})$ and reliability function $R(\underline{p})$ of a $n$-component series system is given by,

$\phi(\underline{x})=\prod_{i=1}^{n} x_{i} \quad$ and $R(\underline{p})=\prod_{i=1}^{n} p_{i}$.

The B-importance of $i^{\text {th }}$ component is,

$I_{B}^{i}(\underline{p})=\prod_{j=1, j \neq i}^{n} p_{j}=\prod_{j=1}^{n} p_{j} / p_{i}$

Thus, the most (least) reliable component has the smallest (largest) B-importance.

Example 2: A parallel system, functions (fails) if and only if at least one (all) component functions (fails). The structure and reliability function for this $n$-component system is given by,

$\phi(\underline{x})=1-\prod_{i=1}^{n}\left(1-x_{i}\right) \quad$ and $\quad R(\underline{p})=1-\prod_{i=1}^{n}\left(1-p_{i}\right)$.

The B-importance of $i^{t h}$ component of this system is,

$I_{B}^{i}(\underline{p})=\prod_{j=1, j \neq i}^{n} q_{j}=\prod_{j=1}^{n} q_{j} / q_{i} ;\left(q_{i}=1-p_{i}\right)$.

That is, the $i^{\text {th }}$ component with the greatest reliability has the highest B-importance (i.e. most reliable component is most important).

Example 3: A $k$-out-of- $n$ system functions (fails) if and only if at least $k(1 \leq k \leq n)$ of the $n$ components function (fail). If $k=n$, the $k$-out-of- $n$ system reduces to series system and if $k=1$, it reduces to the parallel system.

The structure function of $n$-component $k$-out-of- $n$ system is,

$\phi(\underline{x})=\left\{\begin{array}{l}1 \text { if } \sum_{i=1}^{n} x_{i} \geq k \\ 0 \text { if } \sum_{i=1}^{n} x_{i}<k\end{array}\right.$

For a $k$-out-of- $n$ system, Kuo and Zhu (2012c) observed that, 
International Journal of Mathematical, Engineering and Management Sciences

Vol. 2, No. 3, 150-171, 2017

https://dx.doi.org/10.33889/IJMEMS.2017.2.3-014

$$
\begin{array}{ll} 
& \phi\left(1_{i}, \underline{x}\right)-\phi\left(0_{i}, \underline{x}\right)=1 \\
\Leftrightarrow \quad & \phi\left(1_{i}, \underline{x}\right)=1 \text { and } \phi\left(0_{i}, \underline{x}\right)=0 \\
\Leftrightarrow \quad & \text { exactly } k-1 \text { of the } n-1 \text { components (excluding component } i \text { ) function. }
\end{array}
$$

Now, B-importance of $i^{\text {th }}$ component for $k$-out-of- $n$ system is

$I_{B}^{i}(\underline{p})=\sum p_{i_{1}} p_{i_{2}} \ldots p_{i_{k-1}}\left(1-p_{i_{k}}\right)\left(1-p_{i_{k+1}}\right) \cdots\left(1-p_{i_{n-1}}\right)$

where the sum is extended over all permutations $\left(i_{1}, i_{2}, \ldots, i_{n-1}\right)$ of the subscripts $\{1,2, \ldots, i-$ $1, i+1, \ldots n\}$.

Further, for $n>2$ and $k \geq 1$, assuming $p_{1} \leq p_{2} \leq \cdots \leq p_{n}$, Boland and Proschan (1983) and Chadjiconstantinidis and Koustras (1999) proved the following.

$$
\begin{aligned}
& p_{i} \geq \frac{k-1}{n-1} \text { for } i=1,2, \ldots, n, \Rightarrow I_{B}^{1}(\underline{p}) \leq I_{B}^{2}(\underline{p}) \leq \cdots \leq I_{B}^{n}(\underline{p}), \\
& p_{i} \leq \frac{k-1}{n-1} \text { for } i=1,2, \ldots, n, \Rightarrow I_{B}^{1}(\underline{p}) \geq I_{B}^{2}(\underline{p}) \geq \cdots \geq I_{B}^{n}(\underline{p}) .
\end{aligned}
$$

\section{- Review of B-Importance of Consecutive-Systems}

A linear consecutive-k-out-of-n: F (C(k, n: F)) system is introduced by Kontoleon (1980), and fails if and only if at least $\mathrm{k}$ consecutive components in the system fail. Zuo (1993) studies the Bimportance of $\mathrm{C}(\mathrm{k}, \mathrm{n}: \mathrm{F})$ and $\mathrm{C}(\mathrm{k}, \mathrm{n}: \mathrm{G})$ system and claimes that the comparison of B-importance between two components for a $\mathrm{C}(\mathrm{k}, \mathrm{n}: \mathrm{G})$ system is the same as that for the $\mathrm{C}(\mathrm{k}, \mathrm{n}: \mathrm{F})$ system. Hwang et al. (2000) showed that the comparison given by Zuo is not correct and provided a correct relation between the B-importance of components of the two systems. Chang et al. (1999) studied the B-importance of the C(k, n: F) system. Chadjiconstantinidis and Koutras (1999) studied the Bimportance of components for the wide class of Markov Chain Imbeddable Systems (MIS) and provided formulae for the evaluation of the B-importance of the components of an MIS through products of transition probability matrices and multiple recurrence relations. Chang et al. (2002) introduced the concept of uniform, half-line and combinatorial B-importance of components for consecutive-k systems. Meng (1996) compares the importance of system components by the structural characteristics of the system. Kamalja (2012) studies the B-importance of consecutivetype systems as $\mathrm{C}(\mathrm{k}, \mathrm{n}: \mathrm{F}), \mathrm{m}$-consecutive-k-out-of- $\mathrm{n}: \mathrm{F}$ and $\mathrm{r}$-within-consecutive-k-out-of-n: $\mathrm{F}$ system. Shen and Cui (2015) studied B-importance for sparsely connected circular consecutive-k systems. Lin et al. (2016) presented the reliability modeling on consecutive- $\mathrm{k}_{\mathrm{r}}$-out-of- $\mathrm{n}_{\mathrm{r}}$ : $\mathrm{F}$ linear zigzag structure and circular polygon structured systems. Cai et al. (2016) proposed a Bimportance-based genetic algorithm to search the near global optimal solution for linear consecutive- $k$-out-of- $n$ system.

\section{(b) Improvement Potential Importance}

The B-importance of $i^{\text {th }}$ component for the system functioning is also known as improvement potential importance of $i^{\text {th }}$ component (Hoyland and Rausand, 1994; Aven and Jensen, 1999; Freixas and Pons, 2008) or risk achievement importance (van der Borst and Schoonakker, 2001). 
International Journal of Mathematical, Engineering and Management Sciences

Vol. 2, No. 3, 150-171, 2017

https://dx.doi.org/10.33889/IJMEMS.2017.2.3-014

$I_{B S}^{i}\left(1_{i}, p\right)$ is the increase in systems' reliability when component $i$ is perfect i.e. when $p_{i}=1$ and represents the maximum potential improvement in systems' reliability that can be obtained by improving the reliability of component $i$.

The improvement potential importance $I_{I P}^{i}(S)$ of the $i^{\text {th }}$ component of the system $S$ is the difference between system reliability with perfect component $i$ (i.e. component with its reliability $\left.p_{i}=1\right)$ and the system reliability with actual component $i$.

That is,

$$
\begin{array}{r}
I_{I P}^{i}(S)=R(S \mid \text { component } i \text { is perfect })-R(S) \\
=P\left(\phi(\underline{x})=1 \mid X_{i}=1\right)-P(\phi(\underline{x})=1) .
\end{array}
$$

\section{(c) Criticality Reliability Importance}

Kuo and Zuo (2003) proposed the criticality reliability importance measure for system failure (functioning) and is the probability that component $i$ fails (functions) and is critical for system failure (functioning) given that the system fails (functions).

The criticality reliability importance $I_{C f}^{i}(S)$ of the $i^{t h}$ component of system failure given that the system fails is defined as follows.

$$
\begin{aligned}
I_{C f}^{i}(S) & =\frac{P\left(X_{i}=0\right)}{P(\phi(\underline{x})=0)}\left(R\left(1_{i}, \underline{p}\right)-R\left(0_{i}, \underline{p}\right)\right) \\
& =\frac{P\left(X_{i}=0\right)}{1-R(S)} I_{B}^{i}(S) .
\end{aligned}
$$

The criticality reliability importance $I_{C S}^{i}(S)$ of the $i^{\text {th }}$ component of system $(S)$ functioning given that the system functions is defined as follows.

$$
\begin{aligned}
I_{C S}^{i}(S) & =\frac{P\left(X_{i}=1\right)}{P(\phi(\underline{x})=1)}\left(R\left(1_{i}, \underline{p}\right)-R\left(0_{i}, \underline{p}\right)\right) \\
& =\frac{P\left(X_{i}=1\right)}{R(S)} I_{B}^{i}(S) .
\end{aligned}
$$

\section{(d) Bayesian Reliability Importance}

Birnbaum (1969) proposes the Bayesian reliability importance, which is defined as the probability that component $i$ fails given that the system fails. Thus the Bayesian importance $I_{\text {Bay }}^{i}(S)$ of the $i^{\text {th }}$ component of system $S$ with structure function $\phi(\underline{x})$ is defined as follows.

$$
\begin{aligned}
I_{\text {Bay }}^{i}(S) & =P\left(X_{i}=0 \mid \phi(\underline{x})=0\right) \\
& =\frac{P\left(\phi(\underline{x})=0 \mid X_{i}=0\right) P\left(X_{i}=0\right)}{P(\phi(\underline{x})=0)} .
\end{aligned}
$$

Kuo and Zhu (2012c) provided a simplified formula for Bayesian importance of $n$-component system $S$ with independent component reliabilities $p_{1}, p_{2}, \ldots, p_{n}$ as follows. 
International Journal of Mathematical, Engineering and Management Sciences

Vol. 2, No. 3, 150-171, 2017

https://dx.doi.org/10.33889/IJMEMS.2017.2.3-014

$I_{\text {Bay }}^{i}(S)=q_{i}\left\{1+\frac{p_{i}}{1-R(\underline{p})} I_{B}^{i}(S)\right\}, \quad i=1,2, \ldots, n$,

where, $I_{B}^{i}(S)$ is B-importance of $i^{\text {th }}$ component of system $S$.

\section{(e) Fussell-Vesely (FV) Importance}

Fussell and Vesely (1972) introduced a component importance measure using cut set and path set and is referred as FV-cut-importance and FV-path-importance respectively.

The FV-cut-importance (path-importance) of component $i$, denoted by $I_{F V^{c}}^{i}(\underline{p})$ is defined as the probability that a component state vector has corresponding cut (path) that causes (makes) system failure (function), and contains a minimal cut in $\overline{\mathcal{C}}_{i}$ (path in $\overline{\mathcal{P}}_{i}$ ) i.e. containing component $i$. Mathematically,

$$
\begin{aligned}
I_{F V^{c}}^{i}(\underline{p}) & =P\left\{\exists C \in \overline{\mathcal{C}}_{i} \text { such that } C \subseteq N_{0}(\underline{X}) \mid \phi(\underline{X})=0\right\} \\
& =\frac{q_{i} P\left\{\left(0_{i}, \underline{x}\right): \exists C \in \overline{\mathcal{C}}_{i} \ni X_{j}=0 \forall j \in C\right\}}{1-R(\underline{p})}
\end{aligned}
$$

and

$$
\begin{aligned}
I_{F V}^{i} p(\underline{p}) & =P\left\{\exists P \in \overline{\mathcal{P}}_{i} \text { such that } P \subseteq N_{1}(\underline{X}) \mid \phi(\underline{X})=1\right\} \\
& =\frac{p_{i} P\left\{\left(0_{i}, \underline{x}\right): \exists P \in \overline{\mathcal{P}}_{i} \ni X_{j}=0 \forall j \in P\right\}}{R(\underline{p})} .
\end{aligned}
$$

We demonstrate it for 2-component series system through following example.

Example 4: Consider a series system with two components with independent reliabilities, $p_{1}=$ 0.98 and $p_{2}=0.96$. The reliability of the series system is,

$R(\underline{p})=p_{1} p_{2}=0.0592$

There is only one cut set containing component 1 and 2, i.e. $\overline{\mathcal{C}}=\{(1,2)\}$.

Now, FV-cut-importance of component 1 and 2, is as follows.

$I_{F V}^{c}(1)=\frac{1-p_{1}}{1-R(\underline{p})}=\frac{1-p_{1}}{1-p_{1} p_{2}}=0.3378$

and

$I_{F V}^{c}(2)=\frac{1-p_{2}}{1-R(\underline{p})}=\frac{1-p_{2}}{1-p_{1} p_{2}}=0.6757$.

This ranking agrees with the ranking of B-importance measure. The weakest component in a series system is the most important.

Example 5: Consider a $n$-component parallel system. For this system constitutes a minimal cut set is $\overline{\mathcal{C}}=\{1,2, \ldots, n\}$. Hence, FV cut-importance for all components is same. A weakness of this measure is that the same level of importance is calculated for all components in a parallel system regardless of their reliabilities. 
International Journal of Mathematical, Engineering and Management Sciences

Vol. 2, No. 3, 150-171, 2017

https://dx.doi.org/10.33889/IJMEMS.2017.2.3-014

\section{Lifetime Importance Measures}

The lifetime importance measures depend on both, the position of the component in the system and component lifetime distribution. A lifetime importance measures are divided into two subclasses as Time-Dependent Lifetime (TDL) importances and Time Independent Lifetime (TIL) importances, depending on whether they are a function of time (Kuo and Zhu, 2012c). A TDL importance evaluates the importance of components at any time and the rankings of component importance induced may vary with time. While TIL importance takes into account the component lifetime over the long term, as such, perhaps gives a more global view of component importance. Every type of reliability importance measure can be transformed to a corresponding TDL importance measure by substituting $\bar{F}_{i}(t)$ (reliability distribution of component $i$ ) for $p_{i}, i=$ $1,2, \ldots, n$, without changing the probabilistic interpretation of the importance measure. Here we discuss some of the lifetime importance measures. Consider the following notations needed to define lifetime importance measures.

$X_{i}(t) \quad$ state of $i^{\text {th }}$ component at time $t$

$\underline{X}(t)=\left(X_{1}(t), X_{2}(t), \ldots, X_{n}(t)\right)$ : vector of states of $n$ components at time $t$

$F_{i}(t) \quad$ lifetime distribution of $i^{\text {th }}$ component

$\bar{F}_{i}(t)=1-F_{i}(t)=E\left[X_{i}(t)\right]$ : reliability of component $i$ at time $t$

$\bar{F}(t)=\left(\bar{F}_{1}(t), \bar{F}_{2}(t), \ldots, \bar{F}_{n}(t)\right)$ : reliability vector of $n$-components at time $t$

$R(\underline{F}(t))=P\{\phi(\underline{X}(t))=1\}=E[\phi(\underline{X}(t))$

\section{(a) Birnbaum Lifetime (B-Lifetime) Importance}

Lambert (1975) and Natvig (1979) extended the B-importance to the B-lifetime importance at time $t$ by using component lifetime distribution.

The B-lifetime importance of component $i$ at time $t$ is denoted by $I_{B}^{i}(\underline{\bar{F}}(t))$ and is defined as the probability that the system is in a state at time $t$ in which component $i$ is critical for the system. That is, the probability that at time $t$ the failure and functioning of component $i$ coincides with system failure and functioning respectively.

Mathematically,

$$
\begin{aligned}
I_{B}^{i}(\underline{\bar{F}}(t)) & =P\left\{\phi\left(1_{i}, \underline{X}(t)\right)-\phi\left(0_{i}, \underline{X}(t)\right)=1\right\} \\
& =R\left(1_{i}, \underline{\bar{F}}(t)\right)-R\left(0_{i}, \underline{\bar{F}}(t)\right) .
\end{aligned}
$$

Xie (1987) provides the following properties for the system with structure function $\phi$, lifetime distribution $F_{\phi}(t)$ and reliability distribution $\bar{F}_{\phi}(t)$ at time $t$ in which a component is in series or parallel with the rest of a system.

- Xie (1988) gives an upper bound for the B-lifetime importance for coherent system with structure function $\phi$ as $I_{B}^{i}(\underline{\bar{F}}(t)) \leq \min \left\{\frac{\bar{F}_{\phi}(t)}{\bar{F}_{i}(t)}, \frac{F_{\phi}(t)}{F_{i}(t)}\right\}$.

- If component $i$ is in series with the rest of a system, then $I_{B}^{i}(\underline{F}(t))=\frac{\bar{F}_{\phi}(t)}{\bar{F}_{i}(t)}$.

- If component $i$ is in series with the rest of a system, then $I_{B}^{i}(\underline{\bar{F}}(t))=\frac{F_{\phi}(t)}{F_{i}(t)}$. 
International Journal of Mathematical, Engineering and Management Sciences

Vol. 2, No. 3, 150-171, 2017

https://dx.doi.org/10.33889/IJMEMS.2017.2.3-014

\section{(b) Criticality Lifetime Importance}

Lambert (1975) defines the criticality lifetime importance for system failure at time $t$, which is relevant to failure diagnosis. The criticality lifetime importance for the system failure of component $i$ at time $t$ is denoted by $I_{C f}^{i}(\underline{F}(t))$ and is defined as, the probability that component $i$ has failed by time $t$ and component $i$ is critical for the system at time $t$, given that the system has failed by time $t$.

Mathematically,

$$
\begin{aligned}
I_{C f}^{i}(\underline{\bar{F}}(t)) & =P\left\{\phi\left(1_{i}, \underline{X}(t)\right)-\phi\left(0_{i}, \underline{X}(t)\right)=1 \text { and } X_{i}(t)=0 \mid \phi(\underline{X}(t)=0)\right\} \\
& =\frac{F_{i}(t)}{1-R(\underline{\bar{F}}(t))} I_{B}^{i}(\underline{\bar{F}}(t)) .
\end{aligned}
$$

Similarly, the criticality lifetime importance for system functioning can be defined.

\section{(c) Fussell-Vesely (FV) Lifetime Importance}

Fussell and Vesely (1972) introduced a component importance measure using minimal cut/path set. Lambert (1975) defines the FV-lifetime cut-importance through a minimal cut set and Kuo and Zhu (2012c) extended the FV-lifetime cut-importance to the FV-lifetime path-importance using a minimal path set.

The FV-lifetime cut-importance of component $i$ at time $t$ is denoted by $I_{F V^{c}}^{i}(\underline{F}(t))$ and is defined as the probability that at least one minimal cut containing component $i$ fails at time $t$ given that the system fails at time $t$. That is,

$$
\begin{aligned}
I_{F V^{c}}^{i}(\underline{\bar{F}}(t)) & =P\left\{\exists C \in \overline{\mathcal{C}}_{i} \text { such that } X_{j}(t)=0 \forall j \in C \mid \phi(\underline{X}(t)=0)\right\} \\
& =\frac{P\left\{\exists C \in \overline{\mathcal{C}}_{i} \text { such that } X_{j}(t)=0 \forall j \in C\right\}}{1-R(\overline{\underline{F}}(t))} .
\end{aligned}
$$

Similarly, the FV lifetime path-importance of component $i$ at time $t$ is denoted by $I_{F V}^{i}(\underline{F}(t))$ and is defined as,

$$
\begin{aligned}
I_{F V}^{i} p(\bar{F}(t)) & \left.=P\left\{\exists P \in \overline{\mathcal{P}}_{i} \text { such that } X_{j}(t)=0 \forall j \in P \mid \phi(\underline{X}(t))=0\right)\right\} \\
& =\frac{P\left\{\exists P \in \overline{\mathcal{P}}_{i} \text { such that } X_{j}(t)=0 \forall j \in P\right\}}{R(\underline{\bar{F}}(t))} .
\end{aligned}
$$

\section{(d) Barlow-Proschan (BP) Lifetime Importance}

Barlow and Proschan (1975) introduced a new importance measure assuming that the reliabilities of all components of the system are $s$-independent and component $i$ have lifetime distribution $F_{i}(t), i=1,2, \ldots, n$ at time $t$.

The BP-lifetime importance of component $i$ at time $t$ is denoted by $I_{B P}^{i}(\underline{F}(t))$ and is defined as the probability that component $i$ is critical for the system over an infinite mission time. That is, 
International Journal of Mathematical, Engineering and Management Sciences

Vol. 2, No. 3, 150-171, 2017

https://dx.doi.org/10.33889/IJMEMS.2017.2.3-014

$I_{B P}^{i}(\underline{\bar{F}}(t))=\int_{0}^{\infty}\left[R\left(1_{i}, \underline{\bar{F}}(t)\right)-R\left(0_{i}, \underline{\bar{F}}(t)\right)\right] d F_{i}(t) \quad i=1,2, \ldots, n$.

$I_{B P}^{i}(\bar{F}(t))$ can be expressed in terms of B-lifetime importance as follows.

$I_{B P}^{i}(\underline{\bar{F}}(t))=\int_{0}^{\infty} I_{B}^{i}(\underline{\bar{F}}(t)) d F_{i}(t)$.

Thus, $I_{B P}^{i}(\underline{\bar{F}}(t))$ is a weighted average of the $I_{B}^{i}(\underline{\bar{F}}(t))$, over an infinite mission time.

Boland and El-Neweihi (1995) provided a precise justification that $I_{B P}^{i}(\bar{F}(t))$ is exactly the probability that the system lifetime coincide with the life of component $i$, that is

$I_{B P}^{i}(\underline{F}(t))=P\left\{T_{\phi}=T_{i}\right\}$

where $T_{\phi}$ is lifetime of system with structure function $\phi$ and

$T_{i}$ is lifetime of component $i$

Iyer (1992) extends the Barlow-Proschan index to the more general case when the component lifetimes are jointly absolutely continuous but not necessarily independent. In this setting the index $I_{B P}$ may depend not only on the structure function $\phi$ but also on the distribution function $F$.

\section{Properties of BP-Lifetime Importance Measure}

a) $0 \leq I_{B P}^{i}(\underline{\bar{F}}(t)) \leq 1$

b) $\sum_{i=1}^{n} I_{B P}^{i}(\bar{F}(t))=1$

c) If $n \geq 2$ and the intersection of supports $F_{j}(t)=P\left(T_{j}<t\right) ;(j=1,2, \ldots n)$ has positive probability with respect to the product distribution $\prod_{j=1}^{n} F_{j}(t)$ then $0<I_{B P}^{i}(\underline{\bar{F}}(t))<1$.

BP-lifetime importance shows that when a component $i$ is in series (parallel) with the rest of the system and it is stochastically the weakest (strongest) component, then $I_{B P}^{i}(\underline{F}(t)) \geq I_{B P}^{j}(\bar{F}(t))$ for all $i \neq j$.

\section{(e) Natvig Importance}

Natvig (1979) introduces a new component importance measure known as Natvig's measure. A Natvig's measure has the same characteristics as the FV-lifetime importance.

If $Z_{i}$ is the reduction in remaining lifetime due to the failure of the $i^{\text {th }}$ component then a Natvig measure of importance $I_{N}^{i}$ of component $i$ is defined as,

$I_{N}^{i}=E\left(Z_{i}\right) / \sum_{j=1}^{n} E\left(Z_{j}\right)$,

Xie (1988) extends the relationship of BP-lifetime measure to the Natvig measure as, 
International Journal of Mathematical, Engineering and Management Sciences

Vol. 2, No. 3, 150-171, 2017

https://dx.doi.org/10.33889/IJMEMS.2017.2.3-014

$I_{B P}^{i}(\underline{\bar{F}}(t)) \geq I_{B P}^{j}(\underline{\bar{F}}(t))$ for all $i \neq j$, then $I_{N}^{i} \geq I_{N}^{j}$ for all $i \neq j$.

Aven (1986) compares $I_{B P}^{i}(\bar{F}(t))$ and $I_{N}^{i}$ by restricting attention to a finite interval of time assuming component lifetimes Weibull distributed. Calculation of $I_{N}^{i}$ is quite feasible for series and parallel systems where proportional hazard modeling for components is appropriate.

\section{Other Importance Measures}

In this section, we overview some other importance measures which are apart from the 3-classes defined by Birnbaum (1969) and briefly discuss it.

\section{(a) Hwang Index}

Hwang (2001) proposes a new index of component importance based on the cut set, path set, cut and path absoluteness and minimal cut and minimal path set.

Hwang index for component $i$ is denoted by $I_{i}^{h}$ and is defined as,

$I_{i}^{h}=\left\{\left|\mathcal{C}_{i}(d)\right|\right\}, \quad d=1,2, \ldots$

where $\mathcal{C}_{i}(d)$ is the set of cut sets of cardinality $d$ that contains component $i$.

\section{(b) Risk Achievement Worth}

Risk Achievement Worth (RAW) has been introduced by Cheok et al. (1998a, 1998b) for risk analysis terminology. Risk Achievement Worth mainly used as a risk importance measure in probabilistic safety assessments of nuclear power stations.

RAW of component $i$ at time $t$ is denoted by $I_{i}^{R A W}(t)$ and defined as the ratio of the system unreliability with component $i$ failed at time $t$ to the actual system unreliability at time $t$. Mathematically,

$I_{i}^{R A W}(t)=\frac{1-R\left(0_{i}, \underline{p}(t)\right)}{1-R(\underline{p}(t))}$.

\section{(c) Risk Reduction Worth}

Cheok et al. (1998a, 1998b) introduced Risk Reduction Worth (RRW) by using risk analysis terminology. RRW of component $i$ at time $t$ is denoted by $I_{i}^{R R W}(t)$ and defined as the ratio of the actual system unreliability to the system unreliability with component $i$ replaced by a perfect component i.e. $p_{i}(t) \equiv 1$.

Mathematically,

$I_{i}^{R R W}(t)=\frac{1-R(\underline{p}(t))}{1-R\left(1_{i}, \underline{p}(t)\right)}$.

\section{(d) Failure Criticality Index}

A Failure Criticality Index (FCI) is introduced by Wang et al. (2004). FCI is a relative index showing the percentage of times that a failure of the component caused a system failure. The FCI of $i^{\text {th }}$ component at time $t$ is denoted by $I_{i}^{F C I}(t)$ and is defined as, 
International Journal of Mathematical, Engineering and Management Sciences

Vol. 2, No. 3, 150-171, 2017

https://dx.doi.org/10.33889/IJMEMS.2017.2.3-014

$I_{i}^{F C I}(t)=\frac{\text { Number of system failures caused by component } i \text { in }(0, t)}{\text { Number of system failures in }(0, t)}$.

\section{(e) Restore Criticality Index}

Restore Criticality Index (RCI) is introduced by Wang et al. (2004) for the repairable system. RCI of component $i$ is the percentage of times that system restoration results from the restoration of component $i$ in time interval $(0, t)$.

The RCI of $i^{t h}$ component at time $t$ is denoted by $I_{i}^{R C I}(t)$ and defined as,

$I_{i}^{R C I}(t)=\frac{\text { Number of actions on component } i \text { that restored the system in }(0, t)}{\text { Number of times the system was restored in }(0, t)}$.

This index gives the percentage of times that a restoration of the component will result in restoring the system from a down state in time interval $(0, t)$.

\section{(f) Operational Criticality Index}

Operational Criticality Index (OCI) has been introduced by Wang et al. (2004). OCI is the percentage of component's down time over the system downtime or the percentage of a component's up time over the system up time.

The OCI of $i^{t h}$ component at time $t$ is denoted by $I_{i}^{O C I}(t)$ and defined as,

$I_{i}^{O C I}(t)=\frac{\text { Total down time of component } i \text { when the system is down in }(0, t)}{\text { Total system down time in }(0, t)}$,

Or equivalently,

$I_{i}^{O C I}(t)=\frac{\text { Total up time of component } i \text { when the system is up in }(0, t)}{\text { Total system up time in }(0, t)}$.

\section{Generalized (Pairs and Group of Components) Importance Measures}

In this section, we discuss the generalized or pairs/group of importance measures and also discuss conditional reliability importance introduced in the literature.

The importance measures that are presented in Section 2 to Section 5, evaluate the strength of an individual component. Here, we discuss importance measures for a pair or group of components, considering the effects and higher order interactions of components on system performance. It includes Joint Reliability Importance (JRI) and Joint Failure Importance (JFI) introduced by Hong and Lie (1993). Note that the order of components does not matter for any importance measure of the pair or the group.

\section{(a) JRI and JFI of Two Components}

JRI (JFI) of two components is a measure of interaction of two components in a system contribution to the system reliability (failure). 
International Journal of Mathematical, Engineering and Management Sciences

Vol. 2, No. 3, 150-171, 2017

https://dx.doi.org/10.33889/IJMEMS.2017.2.3-014

Mathematically, JRI of component $c_{1}, c_{2}$ is denoted by $J R I\left(c_{1}, c_{2}\right)$ and for $n$-component system with reliability function $R(p)$ of statistically independent component reliabilities $p_{1}, p_{2}, \ldots, p_{n}$ is defined as,

$J R I\left(c_{1}, c_{2}\right)=\frac{\partial^{2} R(\underline{p})}{\partial p_{1} \partial p_{2}}$

While JFI of $c_{1}, c_{2}$ is denoted by $\operatorname{JFI}\left(c_{1}, c_{2}\right)$ and for a system with statistically independent component reliabilities is defined as,

and

$J F I\left(c_{1}, c_{2}\right)=\frac{\partial^{2} F(q)}{\partial q_{1} \partial q_{2}}$

Armstrong (1995) extends the concept of JRI to include a system with $s$-dependent components and proved that JRI is always non-zero for some classes of systems.

Example 6: Consider the $n$-component series system $\left(S_{1}\right)$ and parallel system $\left(S_{2}\right)$ with $s$ independent component reliabilities $p_{1}, p_{2}, \ldots, p_{n}$.

$$
\begin{array}{ll}
R\left(S_{1}\right)=\prod_{i=1}^{n} p_{i}, & R\left(S_{2}\right)=1-\prod_{i=1}^{n}\left(1-p_{i}\right), \\
J R I_{u_{1}, u_{2}}\left(S_{1}\right)=\prod_{i=3}^{n} p_{i}>0, & J R I_{u_{1}, u_{2}}\left(S_{2}\right)=-\prod_{i=1}^{n}\left(1-p_{i}\right)<0 .
\end{array}
$$

\section{- Brief Review of JRI of k-Out-of-n: G System}

Hong et al. (2002) identified the sign of the JRI of two components without finding its value using Schur-convexity of reliability function and studied JRI for k-out-of-n: G system. Hong et al. (2002) compared the JRI of 2-out-of-n and 2-out-of- $(n+1)$ system and stated the inequality about JRI of pair of components as

$J R I_{2, n}(p)<J R I_{2, n+1}(p)$ when $p>\frac{1}{2}$.

where, $J R I_{2, n}(p)$ is the JRI of $c_{1}, c_{2}$ of 2-out-of- $n$ system with i.i.d. component reliabilities $p$.

The formula for JRI of 2-components of the $k$-out-of- $n$ system with i.i.d. component reliabilities for $n \geq 3$ and $2 \leq k \leq n$ given by Hong et al. (2002) is as follows.

$J R I_{k, n}\left(c_{i}, c_{j}\right)=p^{k-2}(1-p)^{n-k-1}\left[\left(\begin{array}{c}n-2 \\ k-2\end{array}\right)-\left(\begin{array}{c}n-1 \\ k-1\end{array}\right) p\right]$.

Hong et al. (2002) stated the results about the signs of JRI of a $k$-out-of- $n$ system with i.i.d. component reliabilities for $n \geq 3$ and $2 \leq k \leq n$ and are as follows.
i) $J R I_{k, n}(p)>0$ if $0<p<\frac{k-1}{n-1}$.
ii) $J R I_{k, n}(p)=0 \quad$ if $p=\frac{k-1}{n-1}$.
iii) $J R I_{k, n}(p)<0 \quad$ if $\frac{k-1}{n-1}<p<1$. 
International Journal of Mathematical, Engineering and Management Sciences

Vol. 2, No. 3, 150-171, 2017

https://dx.doi.org/10.33889/IJMEMS.2017.2.3-014

From above results, it can be seen that a system with i.i.d. component reliabilities $p$, the JRI of two components is positive for smaller values of $p$ and negative for larger values of $p$. Moreover the threshold is $p=\frac{k-1}{n-1}$.

Chang and Jan (2006) re-state the properties of JRI of the 2-out-of-n system and correct the errors of Hong et al. (2002). The properties are as follows.

i) $J R I_{2, n}(p)=(1-p)^{n-3}[1-(n-1) p]$.

ii) $\lim _{p \rightarrow 0} J R I_{2, n}(p)=1$ and $\lim _{p \rightarrow 1} J R I_{2, n}(p)=0$.

iii) $\operatorname{Min}\left\{J R I_{2, n}(p)\right\}=-\left(\frac{n-3}{n-1}\right)^{n-3}$ when $p=\frac{2}{n-1}$.

iv) For $n \geq 5$, the graph of $J R I_{2, n}(p)$ has a point of inflection at $\left(\frac{3}{n-1},-2\left(\frac{n-4}{n-1}\right)^{n-3}\right)$.

\section{- JRI of Three Components}

The JRI of three components $c_{1}, c_{2}$ and $c_{3}$ for $n$-component system with reliability function $R(p)$ and component reliabilities $p_{1}, p_{2}, \ldots, p_{n}$ is given by,

$J R I\left(c_{1}, c_{2}, c_{3}\right)=\frac{\partial^{3} R(\underline{p})}{\partial p_{1} \partial p_{2} \partial p_{3}}$.

For independent component reliabilities, $J R I\left(c_{1}, c_{2}, c_{3}\right)$ simplifies as follows.

$$
\begin{aligned}
J R I\left(c_{1}, c_{2}, c_{3}\right)= & R\left(1_{1}, 1_{2}, 1_{3}, \underline{p}\right)-R\left(1_{1}, 1_{2}, 0_{3}, \underline{p}\right)-R\left(1_{1}, 0_{2}, 1_{3}, \underline{p}\right) \\
& -R\left(0_{1}, 1_{2}, 1_{3}, \underline{p}\right)+R\left(1_{1}, 0_{2}, 0_{3}, \underline{p}\right)+R\left(0_{1}, 1_{2}, 0_{3}, \underline{p}\right) \\
& +R\left(0_{1}, 0_{2}, 1_{3}, \underline{p}\right)-R\left(0_{1}, 0_{2}, 0_{3}, \underline{p}\right),
\end{aligned}
$$

where, $R\left(l_{1}, l_{2}, l_{3}, \underline{p}\right)$ : Reliabilities of $n$-component system with component reliabilities $\left(p_{1}, p_{2}, \ldots, l_{1}, \ldots, l_{2}, \ldots, l_{3}, \ldots, p_{n}\right)$.

Example 7: The JRI of 3 components for $k$-out-of- $n$ : $G$ system with i.i.d. component reliabilities $p$ and $k \geq 3$ (given by Gao et al. (2007) is,

$J R I\left(c_{1}, c_{2}, c_{3}\right)=p^{k-3} q^{n-k-2}\left(\left(\begin{array}{l}n-3 \\ k-3\end{array}\right) q^{2}-2\left(\begin{array}{l}n-3 \\ k-2\end{array}\right) p q+\left(\begin{array}{l}n-3 \\ k-1\end{array}\right) p^{2}\right)$.

\section{- JRI of More Than Three Components}

The definition of JRI of 3 components to a group of components is extended as follows.

The JRI for $r(r<n)$ components for $c_{1}, c_{2}, \ldots, c_{r}$ is defined as,

$J R I\left(c_{1}, c_{2}, \ldots, c_{r}\right)=\frac{\partial^{r} R(\underline{p})}{\prod_{i=1}^{r} \partial p_{i}}$.

For independent component reliabilities, $J R I\left(c_{1}, c_{2}, \ldots, c_{r}\right)$ simplifies to $J R I\left(c_{1}, c_{2}, \ldots, c_{r}\right)=R\left(1_{1}, 1_{2}, \ldots, 1_{r}, \underline{p}\right)-R\left(1_{1}, 1_{2}, \ldots, 0_{r}, \underline{p}\right) \pm \cdots$ 
International Journal of Mathematical, Engineering and Management Sciences

Vol. 2, No. 3, 150-171, 2017

https://dx.doi.org/10.33889/IJMEMS.2017.2.3-014

$$
\pm R\left(0_{1}, 0_{2}, \ldots, 1_{r}, \underline{p}\right) \pm R\left(0_{1}, 0_{2}, \ldots, 0_{r}, \underline{p}\right)
$$

Zhu et al. (2015) studied the joint reliability importance of consecutive- $k$-out-of- $n: F$ and $m$ consecutive- $k$-out-of- $n: F$ system for Markov dependent components.

\section{(b) Conditional Reliability Importance}

The conditional reliability importance is introduced by Gao et al. (2007). Under the condition that some components are working or some components are failed, the reliability importance of other components is evaluated. Based on the definition of Marginal Reliability Importance (MRI) and JRI, we consider the reliability importance when the states of certain components are known. It is assumed that the system also works under these conditions. Here we present the definitions of Conditional MRI and Conditional JRI given by Gao et al. (2007).

The conditional MRI of component $c_{j}$, when component $c_{i}$ is working or failed (i.e. $p_{i}=z_{i}, z_{i}=$ $0,1)$ is denoted by $M R I_{j}\left(p_{i}=z_{i}\right)$ and is defined as,

$M R I_{j}\left(p_{i}=z_{i}\right)=\frac{\partial R\left(p_{1}, p_{2}, \ldots, p_{i-1}, z_{i}, p_{i+1}, \ldots, p_{n}\right)}{\partial p_{j}}, i, j=1,2, \ldots, n, j \neq i$

For independent component reliabilities, $M R I_{j}\left(p_{i}=z_{i}\right)$ simplifies to

$$
\begin{aligned}
M R I_{j}\left(p_{i}=z_{i}\right)= & R\left(p_{1}, \ldots, z_{i}, \ldots, p_{j-1}, 1_{j}, p_{j+1}, \ldots, p_{n}\right) \\
& -R\left(p_{1}, \ldots, z_{i}, \ldots, p_{j-1}, 0_{j}, p_{j+1}, \ldots, p_{n}\right),
\end{aligned}
$$

where $z_{i}=1(0)$ when the component $c_{i}$ is working (failed).

JRI can be expressed in terms of MRI as follows.

$\operatorname{JRI}\left(c_{i}, c_{j}\right)=M R I_{j}\left(p_{i}=1\right)-M R I_{j}\left(p_{i}=0\right)$.

The conditional JRI of components $c_{i}$ and $c_{j}$, when the state of component $c_{k}$ is denoted by $J R I\left(c_{i}, c_{j}\right)\left(p_{k}=z_{k}\right)$ and is defined as,

$\operatorname{JRI}\left(c_{i}, c_{j}\right)\left(p_{k}=z_{k}\right)=\frac{\partial^{2} R\left(p_{1}, \ldots, z_{k}, \ldots, p_{n}\right)}{\partial p_{i} \partial p_{j}}, k \neq i, j$.

For independent component reliabilities, $J R I\left(c_{i}, c_{j}\right)\left(p_{k}=z_{k}\right)$ simplifies to

$$
\begin{aligned}
J R I\left(c_{i}, c_{j}\right)\left(p_{k}=z_{k}\right)= & R\left(z_{k}, 1_{i}, 1_{j}, \underline{p}\right)-R\left(z_{k}, 1_{i}, 0_{j}, \underline{p}\right)-R\left(z_{k}, 0_{i}, 1_{j}, \underline{p}\right) \\
& +R\left(z_{k}, 0_{i}, 0_{j}, \underline{p}\right)
\end{aligned}
$$

Rani et al. (2011) and Jain et al. (2014) obtained expressions for conditional MRI and JRI when the component reliabilities are independent but need not be identically distributed for series-inparallel and series-parallel systems. 
International Journal of Mathematical, Engineering and Management Sciences

Vol. 2, No. 3, 150-171, 2017

https://dx.doi.org/10.33889/IJMEMS.2017.2.3-014

\section{Review of Importance Measures of Weighted-Systems}

In many engineering systems, its components contribute to the system not only through its (working or fail) status, but also through their capacity/load (weight). The components contribute differently to the system performance. Thus, components of a system may be associated with an additional weight characterization apart from its status. In real life, the weight of a component may be its heating/cooling capacity, voltage/wattage, test-score etc. relative to the whole system. This leads to the concept of weighted systems, which consists of components having some positive integer weight.

$\mathrm{Wu}$ and Chen (1994a) introduced a weighted $k$-out-of- $n: G(F)$ system which consists of $n$ components, each one having positive integer weight $w_{i}$ such that the total system weight is $w=$ $\sum_{i=1}^{n} w_{i}$ and the system works (fail) if and only if the total weight of working (failed) components is at least $k$, a prespecified value. Since $k$ is a weight, it may be larger than $n$. Samaniego and Shaked (2008) referred these types of systems as Systems with Weighted Components (SWCs). Further Wu and Chen (1994b) introduced and studied a weighted version of consecutive- $k$-out-of$n: F\left(C^{w}(k, n: F)\right)$ system in linear as well as circular case. A weighted-consecutive- $k$-out-of- $n: F$ system fails if and only if the total weight of failed consecutive components is at least $k$.

Chadjiconstantinidis and Koutras (1999) provided formulae for the evaluation of the B-importance and the improvement potential importance of components of weighted k-out-of- $n: F$ and $\mathrm{C}^{\mathrm{w}}(\mathrm{k}, \mathrm{n}: \mathrm{F})$ system using Markov chain imbedding technique. Amrutkar and Kamalja (2014) developed formulae for evaluation of reliability and the reliability importance of weighted k-outof-n: Fsystem through Weighted Markov Binomial Distribution (WMBD). Eryilmaz and Bozbulut (2014) studied joint and marginal Birnbaum and Barlow-Proschan importance measures of components of weighted k-out-of-n: $G$ system by using the universal generating function. Rahmani et al. (2016) studied the importance of components in k-out-of-n system with components having random weights. Kamalja and Amrutkar (2014) studied reliability importance of $\mathrm{C}^{\mathrm{w}}(\mathrm{k}, \mathrm{n}: \mathrm{F})$ system.

\section{Discussion and Conclusions}

In this paper we presented an overview of various importance measures of coherent system. Apart from the importance measures discussed in the present manuscript, there are many other importance measures. This paper is an effort to overview the some of the popular importance measures and study of these for some common systems such as, series, parallel, $k$-out-of- $n$, consecutive and their weighted versions.

In this paper, we overviewed the recent literature on component importance measures. Further, we presented these importance measures for different well-known systems. This survey would spread awareness among readers regarding the importance measures available as well as encourage further research in deciding, which importance measures and when it should be used.

\section{Acknowledgement}

(i) This work is supported by UGC, New Delhi, India, through the Major Research Project (F. No. 40246/2011 (SR)).

(ii) The first author is thankful to the CSIR, New Delhi, for awarding Senior Research Fellowship (F. No. 09/728 (0033)/2014-EMR-I).

(iii) The authors would like to thank Guest Editor (s) for their constructive comments that improved the manuscript. 
International Journal of Mathematical, Engineering and Management Sciences

Vol. 2, No. 3, 150-171, 2017

https://dx.doi.org/10.33889/IJMEMS.2017.2.3-014

\section{References}

Amrutkar, K. P., \& Kamalja, K. K. (2014). Reliability and importance measures of weighted- $k$-out-of- $n: F$ system. International Journal of Reliability, Quality and Safety Engineering, 21(03), 1450015.

Armstrong, M. J. (1995). Joint reliability-importance of components. IEEE Transaction on Reliability, 44(3), 408-412.

Aven, T. (1986). On the computation of certain measures of importance of system components. Microelectronics Reliability, 26(2), 279-281.

Aven, T., \& Jensen, U. (1999). Stochastic models in reliability. Springer, New York.

Barlow, R. E., \& Proschan, F. (1975). Importance of system components and fault tree event. Stochastic Processes and their Applications, 3(2), 153-173.

Barlow, R. E., \& Proschan, F. (1975). Statistical theory of reliability and life testing. Holt, Rinheart and Winston, New York.

Bergman, B., Arjas, E., Rausand, M., Natvig, B., Doksum, K. A., \& Schweder, T. (1985). On reliability theory and its applications [with Discussion and Reply]. Scandinavian Journal of Statistics, 1-41.

Birnbaum, Z. W. (1969). On the importance of different components in a multi-component system. Multivariate Analysis-II, P.R. Krishnaiah (Editor), Academic, New York, 581-592.

Boland, P. J., \& El-Neweihi, E. (1995). Measures of component importance in reliability theory. Computers \& Operations Research, 22(4), 455-463.

Boland, P. J., \& Proschan, F. (1983). The reliability of $k$-out-of-n systems. The Annals of Probability, 11(3), 760-764.

Boland, P. J., Proschan, F., \& Tong, Y. L. (1989). Optimal arrangement of components via pairwise rearrangements. Naval Research Logistics, 36, 807-815.

Butler, D. A. (1979). A complete importance ranking for components of binary coherent systems, with extensions to multi-state systems. Naval Research Logistics Quarterly, 26(4), 565-578.

Cai, Z., Si, S., Sun, S., \& Li, C. (2016). Optimization of linear consecutive-k-out-of-n system with a Birnbaum importance-based genetic algorithm. Reliability Engineering \& System Safety, 152, 248-258.

Chadjiconstantinidis, S., \& Koutras, M. V. (1999). Measures of component importance for Markov chain imbeddable reliability structures. Naval Research Logistics (NRL), 46(6), 613-639.

Chang, G. J., Cui, L., \& Hwang, F. K. (1999). New comparisons in Birnbaum importance for the consecutivek-out-of-n system. Probability in the Engineering and Informational Sciences, 13(02), 187-192.

Chang, H. W., \& Jan, S. (2006). Joint reliability importance of $k$-out-of- $n$ systems and series-parallel systems. Proceeding Techniques and Applications \& Conference on Real-Time Computing Systems and Applications, PDPTA, Las Vegas, Nevada, USA.

Chang, H. W., Chen, R. J., \& Hwang, F. K. (2002). The structural Birnbaum importance of consecutive-k systems. Journal of Combinatorial Optimization, 6(2), 183-197.

Cheok, M. C., Parry, G. W., \& Sherry, R. R. (1998a). Response to 'Supplemental viewpoints on the use of importance measures in risk-informed regulatory applications'. Reliability Engineering \& System Safety, 60(3), 261.

Cheok, M. C., Parry, G. W., \& Sherry, R. R. (1998b). Use of importance measures in risk-informed regulatory applications. Reliability Engineering \& System Safety, 60(3), 213-226.

Eryilmaz, S., \& Bozbulut, A. R. (2014). Computing marginal and joint Birnbaum, and Barlow-Proschan importances in weighted-k-out-of-n: G systems. Computers \& Industrial Engineering, 72, 255-260. 
International Journal of Mathematical, Engineering and Management Sciences

Vol. 2, No. 3, 150-171, 2017

https://dx.doi.org/10.33889/IJMEMS.2017.2.3-014

Freixas, J., \& Pons, M. (2008). The influence of the node criticality relation on some measures of component importance. Operation Research Letters, 36(5), 557-560.

Fussell, J. B., \& Vesely, W. E. (1972). A new methodology for obtaining cut sets for fault trees. Transactions of the American Nuclear Society, 15, 262-263.

Gao, X., Cui, L., \& Li, J. (2007). Analysis for joint importance of components in a coherent system. European Journal of Operational Research, 182(1), 282-299.

Hong, J. S., \& Lie, C. H. (1993). Joint reliability-importance of two edges in an undirected network. IEEE Transactions on Reliability, 42(1), 17-23.

Hong, J. S., Koo, H. Y., \& Lie, C. H. (2002). Joint reliability importance of k-out-of-n systems. European Journal of Operational Research, 142(3), 539-547.

Hoyland, A., \& Rousand, M. (1994). System Reliability Theory. New York: John Wiley \& Sons.

Hsu, S. J., \& Yuang, M. C. (1999). Efficient computation of marginal reliability importance for reducible networks in network management. Proceedings of the 1999 IEEE International Conference on Communications, 1039-1045.

Hwang, F. K. (2001). A new index of component importance. Operations Research Letters, 28(2), 75-79.

Hwang, F. K., Cui, L., Chang, J. C., \& Lin, W. D. (2000). Comments on "reliability and component importance of a consecutive-k-out-of-n system" by Zuo. Microelectronics Reliability, 40(6), 1061-1063.

Iyer, S. (1992). The Barlow-Proschan importance and its generalizations with dependent components. Stochastic Processes and their Applications, 42(2), 353-359.

Jain, K., Dewan, I., \& Rani, M. (2014). Multicomponent joint reliability importance of series-in-parallel and parallel-in-series systems. International Journal of Quality \& Reliability Management, 31(7), 858-876.

Kamalja, K. K. \& Amrutkar, K. P. (2014). Computational methods for reliability and importance measures of weighted-consecutive-systems, IEEE Transaction on Reliability, 63(1), 94-104.

Kamalja, K. K. (2012). Birnbaum importance for consecutive-k systems. International Journal of Reliability, Quality and Safety Engineering, 19(4), 1250016 (25 pages).

Kontoleon, J. M. (1980). Reliability determination of a r-successive-out-of-n: F system. IEEE Transactions on Reliability, 29(5), 437-437.

Koutras, M. V., Papadopoylos, G., \& Papastavridis, S. G. (1994). Note: Pairwise rearrangements in reliability structures. Naval Research Logistics, 41, 683-687.

Kuo, W., \& Zhu, X. (2012a). Relations and generalizations of importance measures in reliability. IEEE Transactions on Reliability, 61(3), 659-674.

Kuo, W., \& Zhu, X. (2012b). Some recent advances on importance measures in reliability. IEEE Transactions on Reliability, 61(2), 344-360.

Kuo, W., \& Zhu, X. (2012c). Importance measures in reliability, risk and optimization: principles and applications. John Wiley \& Sons, Chichester, UK. 472.

Kuo, W., \& Zuo, M.J. (2003). Optimal reliability modelling: Principles and Applications. John Wiley \& Sons, New Jersey.

Lambert, H. E. (1975). Measure of importance of events and cut sets in fault trees. Reliability and Fault Tree Analysis (eds. Barlow, R.E., Fussell, J.B. and Singpurwalla, N.D.). Society for Industrial and Applied Mathematics, Philadelphia, 77-100. 
International Journal of Mathematical, Engineering and Management Sciences

Vol. 2, No. 3, 150-171, 2017

https://dx.doi.org/10.33889/IJMEMS.2017.2.3-014

Lin, C., Cui, L., Coit, D. W., \& Lv, M. (2016). Reliability modeling on consecutive-\$ k_r \$-out-of-\$ n_r \$: F linear zigzag structure and circular polygon structure. IEEE Transactions on Reliability, 65(3), 15091521.

Lu, L., \& Jiang, J. (2007). Joint failure importance for noncoherent fault trees. IEEE Transactions on Reliability, 56(3), 435-443.

Meng, F. C. (1996). Comparing the importance of system components by some structural characteristics, IEEE Transactions on Reliability, 45(1), 59-65.

Natvig, B. (1979). A suggestion of a new measure of importance of system components. Stochastic Processes and their Applications, 9(3), 319-330.

Rahmani, R. A., Izadi, M., \& Khaledi, B. E. (2016). Importance of components in k-out-of-n system with components having random weights. Journal of Computational and Applied Mathematics, 296, 1-9.

Rani, M., Jain, K., \& Dewan, I. (2011). On conditional marginal and conditional joint reliability importance. International Journal of Reliability, Quality and Safety Engineering, 18(2), 119-138.

Samaniego, F. J., \& Shaked, M. (2008). Systems with weighted components. Statistics \& Probability Letters, 78(6), 815-823.

Shen, J., \& Cui, L. (2015). Reliability and Birnbaum importance for sparsely connected circular consecutive\$ k \$ systems. IEEE Transactions on Reliability, 64(4), 1140-1157.

Van der Borst, M., \& Schoonakker, H. (2001). An overview of PSA importance measures. Reliability Engineering \& System Safety, 72(3), 241-245.

Wang, W., Loman, J., \& Vassiliou, P. (2004). Reliability importance of components in a complex system, Reprinted from "2004 Proceedings Annual Reliability and Maintainability Symposium," Los Angeles, California, USA, January 26-29.

Wu, J. S., \& Chen, R. J. (1994a). An algorithm for computing the reliability of weighted-k-out-of-n systems. IEEE Transactions on Reliability, 43(2), 327-328.

Wu, J. S., \& Chen, R. J. (1994b). Efficient algorithms for k-out-of-n and consecutive-weighted-k-out-of-n: F system. IEEE Transactions on Reliability, 43(4), 650-655.

Xie, M. (1987). On some importance measures of system components. Stochastic Process and their Applications, 25, 273-280.

Xie, M. (1988). A note on the natvig measure. Scandinavian Journal of Statistics, 15, 211-214.

Zhu, X., \& Kuo, W. (2014). Importance measures in reliability and mathematical programming. Annals of Operations Research, 212(1), 241-267.

Zhu, X., Boushaba, M., \& Reghioua, M. (2015). Joint reliability importance in a consecutive-k-out-of-n: F system and an m-consecutive-k-out-of-n: $\mathrm{F}$ system for Markov-dependent components. IEEE Transactions on Reliability, 64(2), 784-798.

Zuo, M. (1993). Reliability and component importance of a consecutive-k-out-of-n system. Microelectronics Reliability, 33(2), 243-258. 